\title{
Wave Propagation in Fiber Reinforced Composites for Oblique Incidence
}

\author{
R. A. KLINE \\ School of Aerospace \\ Mechanical and Nuclear Engineering \\ The University of Oklahoma \\ Norman, Oklahoma
}

(Received January 4, 1987)

\section{INTRODUCTION}

$\mathbf{U}$ LTRASONIC NONDESTRUCTIVE TESTING has become the method of choice for the quality assurance of advanced composite materials. This inspection usually involves an immersion C-scan procedure with defect identification based on an analysis of the reflected wave amplitudes. This procedure works well for identifying gross composite defects. However, in many cases, one is concerned with more subtle defects (e.g., porosity, local variation in fiber orientation, segregation of reinforcing fibers, etc.); defects which are difficult ot identify with conventional data analysis procedures. Since defects such as these will principally affect the local moduli, ultrasonic velocity measurements are quite useful in analyzing these types of problems [1]. Further, since fiber reinforced composites are anisotropic materials, one would ideally like to examine directional dependence of the properties by measuring all pertinent elastic moduli. This is particularly important for those moduli largely influenced by the fiber reinforcement. Previous investigators have performed the ultrasonic tests required to completely characterize all five elastic moduli for transversely isotropic materials and all nine elastic moduli of orthotropic materials [2-5]. However, this procedure is rarely used in practice for several important reasons.

These tests were all conducted using contact transducers at normal incidence. This approach, while useful for measurements at a given point, is unsuitable for the scanning of large parts due to the difficulty of maintaining shear coupling as the transducer is scanned. An alternative approach is to use immersion transducers with mode conversion to generate the required waves additional to the longitudinal wave as suggested by Hosten and Tittmann [6]. Unfortunately, the mode conversion approach to the generation of waves in an anisotropic media is significantly more complicated than the isotropic case. Since the generated waves do not have their wave normal along symmetry axes, they will not usually be pure mode waves but rather quasilongitudinal or quasitransverse waves. Furthermore, 
as has been observed many times in anisotropic media, the energy flux associated with wave propagation will often deviate from the wave normal complicating the problem considerably. A further problem stems from the variation in wave speed with propagation direction in the composite and the associated problem with determining the angle of refraction via Snell's law. While the approach used by Hosten and Tittmann has several drawbacks, notably in ignoring energy flux considerations and the fact that it can't be used for analytical scanning, the basic ideas is sound and may be implemented providing that the details of wave generation at the interface are understood.

Previous work in this area has been quite limited and principally applied to single crystals, not composites. Musgrave [7] attacked the problem with a graphical solution method which, while useful, is subject to error. More accurate numerical techniques have been developed to address this shortcoming, notably that of Rokhlin et al., who treat the complete problem of reflection and refraction at the interface between two general anisotropic media [8-11]. These treatments are somewhat cumbersome as they must be sufficiently general to handle materials with no structural symmetry whatsoever. This results in a relatively complex formulation of the problem. However, a considerable degree of simplification can be achieved by restricting attention to the special case of a unidirectionally reinforced composite. This also avoids the problem of additional mode conversion at ply boundaries. Ultimately, if this technique is to have practical value, these factors must also be included in the analysis. The principal objective of this work was to develop a simplified method for analyzing reflectionrefraction phenomena in transversely isotropic materials for arbitrary angles of incidence. Ideally, a method was sought which was rapid, accurate and sufficiently compact to be implemented on a laboratory microcomputer so that it would be useful for nondestructive testing purposes.

\section{THEORY}

\section{Slowness Surface for Composites}

Before discussing wave propagation in anisotropic media, it is first useful to review the concepts of slowness vectors and the slowness surface. Defining the slowness vector as

$$
\underline{m}=\frac{1}{\omega} \underline{k}=\frac{1}{v} \underline{\ell}
$$

where

$$
\begin{aligned}
& \underline{k}=\text { wave vector }=|\underline{k}| \underline{\ell} \\
& \underline{k}=|\underline{k}|=\text { wave number } \\
& \underline{\ell}=\text { wave normal }
\end{aligned}
$$

the slowness surface represents the locus of the endpoints of the slowness vectors. For an isotropic medium, the slowness surface consists of three concentric 


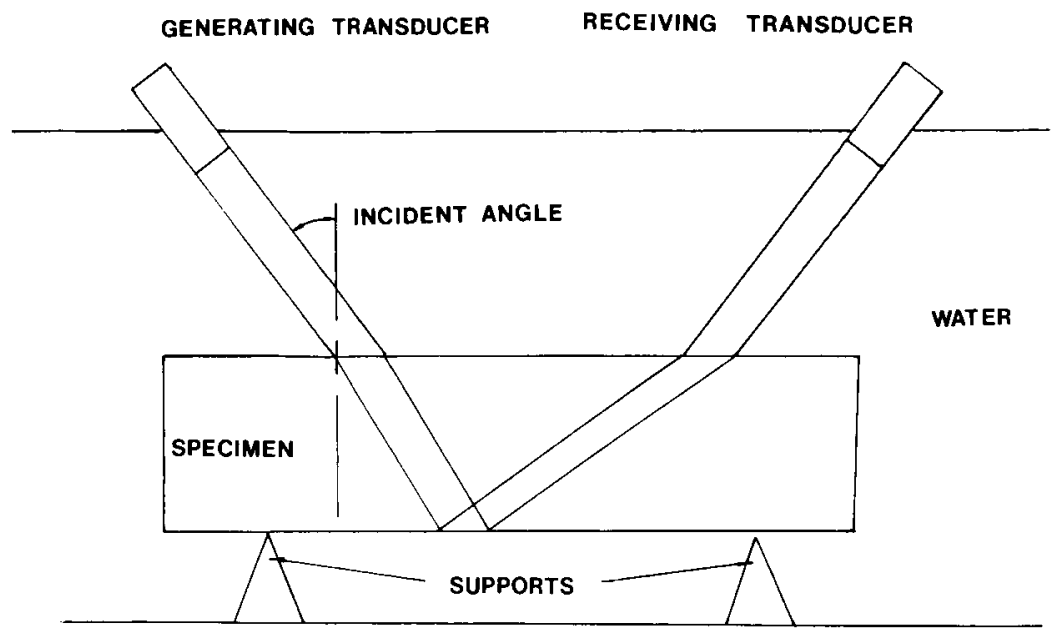

Figure 1. Experimental arrangement.

spherical sheets. The inner sheet then represents the longitudinal wave and the outer sheets are two coincident sheets associated with shear wave propagation. The sheets are spherical because the velocities in an isotropic medium are independent of the propagation direction. The two shear sheets coincide since the two velocities of shear wave propagation are identical. For anisotropic media, there are three distinct sheets of arbitrary shape. The shape of the slowness surface is an important factor in determining the nature of reflected and transmitted waves in anisotropic media as will be shown in the following dimension.

The problem under consideration consists of a plane longitudinal wave in water incident upon the boundary of a unidirectional composite panel. For the cases to be studied, the wave vector for the incident wave lies in a plane either parallel or perpendicular to the fiber reinforcement. The geometry is illustrated in Figure 1. With this geometry, the incident wave may be represented as

$$
\underline{u}_{i n}=\underline{A} e^{i \omega^{i n}\left(m_{k}^{i n} x_{k}-t\right)}
$$

Similarly, the reflected longitudinal wave may be represented as

$$
\underline{u}_{r e}=A_{r e} e^{i \omega r e\left(m_{k}^{r e} x_{k}-t\right)}
$$

For the transmitted waves, we have

$$
\underline{u}^{i}=A_{i} e^{i \omega_{k}^{i}\left(m i x_{k}^{i} x_{k}^{-t)}\right.}
$$

where the superscript $i$ is used to differentiate between the transmitted work. In order to satisfy the boundary conditions at the interface, the frequencies of all 
waves must be equal, i.e.

$$
\omega=\omega^{i n}=\omega^{r e}=\omega^{i}
$$

and

$$
m_{k}^{i n} x_{k}=m_{k}^{r e} x_{k}=m_{k}^{i} x_{k}
$$

Henneke has shown that Equation (5) is equivalent to

$$
a_{i}=\epsilon_{i j k} m_{j}^{i n} \nu_{k}=\epsilon_{i j k} m_{j}^{r e} \nu_{k}=\epsilon_{i j k} m_{j}^{i} \nu_{m}
$$

where

$\underline{\nu}=$ normal to interface

$\underline{a}=$ constant vector quantity

This statement is precisely Snell's law. To see this, let

$$
\underline{\nu}=\left(\begin{array}{l}
1 \\
0 \\
0
\end{array}\right)
$$

and

$$
\underline{\sim}^{i n}=\frac{1}{V_{w}}\left(\begin{array}{c}
\cos \theta^{i n} \\
0 \\
\sin \theta^{i n}
\end{array}\right)
$$

as is appropriate for this geometry so that

$$
\underset{\sim}{a}=\left(\begin{array}{c}
0 \\
\frac{\sin \theta^{i n}}{V_{w}} \\
0
\end{array}\right)
$$

Equation (5) then becomes

$$
\left(\begin{array}{c}
0 \\
\frac{\sin \theta^{i n}}{V_{w_{O}}}
\end{array}\right) \quad\left(\begin{array}{c}
0 \\
m^{r e} \\
-m_{2}^{r e}
\end{array}\right) \quad\left(\begin{array}{c}
0 \\
m^{i} \\
-m_{2}^{i}
\end{array}\right)
$$

This requires

$$
m_{2}^{r e}=m_{2}^{i}=0
$$


Then, since $\ell$ must be a unit vector, we may use the following representation:

$$
\underline{m}^{r e}=\frac{1}{V_{w}}\left(\begin{array}{c}
-\cos \theta_{i n} \\
0 \\
\sin \theta_{i n}
\end{array}\right) \quad \text { and } \quad \underline{m}^{i}=\frac{1}{V_{i}}\left(\begin{array}{c}
\cos \theta^{i} \\
0 \\
\sin \theta^{i}
\end{array}\right)
$$

where the negative sign in the slowness vector of the reflected wave is included to indicate that it is propagating away from the interface and into the water. Then, we have

$$
\frac{\sin \theta^{i n}}{V_{w}}=\frac{\sin \theta^{r e}}{V_{w}}=\frac{\sin \theta^{i}}{V_{i}}
$$

which is, of course, Snell's law. Letting

$$
\underline{b}=\underline{\nu} \times \underline{a}=\left(\begin{array}{c}
0 \\
0 \\
\sin \theta^{i n} \\
\hline V_{w}
\end{array}\right)
$$

the reflection-refraction problem may be represented geometrically by Figure 2 . Here, the slowness surfaces and slowness vectors for the various waves are presented. We seek solutions to the equations of motion such that the projection of

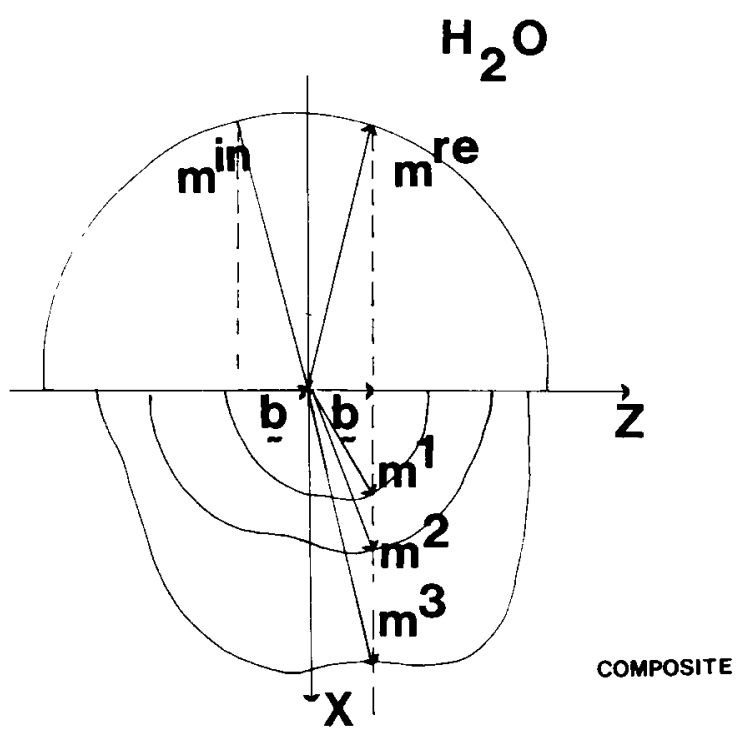

Figure 2. Geometric representation of Snell's law. 
the slowness vector on the boundary $(|\underline{b}|)$ is the same for all waves under consideration. For an isotropic medium, this means that there will be two possible refracted waves. For an anisotropic medium, the number and nature of the refracted waves depend upon the shape of the slowness surface. The usual case, three possible refracted waves, is shown in Figure 2. However, as Henneke demonstrated, other configurations of the slowness surface are possible [9].

\section{Velocity Considerations}

The eigenvalue equation for wave propagation in an anisotropic medium is given by

$$
\left(\underline{\underline{\lambda}}-\varrho V^{2} \underline{z}\right) \underline{\alpha}=0
$$

where

$$
\begin{aligned}
\underline{I} & =\text { identity matrix } \\
\underline{Q} & =\text { density } \\
V & =\text { velocity } \\
\lambda_{i \kappa} & \equiv C_{i j k \ell} \ell_{j} \ell_{l} \\
\underline{\alpha} & =\text { direction cosines of particle displacements } \\
\underline{\ell} & =\text { direction cosines of wave normal }
\end{aligned}
$$

Using the representation of the wave normal for the refracted waves in the orthotropic medium found earlier

$$
\underline{\ell}=\left(\begin{array}{c}
\cos \theta^{i} \\
0 \\
\sin \theta^{i}
\end{array}\right)
$$

we have

$$
\begin{aligned}
\lambda_{11}=C_{1 j 1 \varepsilon} \ell_{j} \ell_{\ell} & =C_{1111} \cos ^{2} \theta^{i}+C_{1311} \cos \theta^{i} \sin \theta^{i} \\
& +C_{1113} \cos \theta^{i} \sin \theta^{i}+C_{1313} \sin ^{2} \theta^{i}
\end{aligned}
$$

which in contracted notation becomes

$$
=C_{11} \cos ^{2} \theta^{i}+C_{55} \sin ^{2} \theta^{i}
$$

Similarly, one can calculate

$$
\begin{aligned}
& \lambda_{12}=C_{1 j} \ell_{i} \ell_{l} \\
& \lambda_{13}=C_{13} \sin \theta^{i} \cos \theta^{i}+C_{55} \sin \theta^{i} \cos \theta^{i}
\end{aligned}
$$




$$
\begin{gathered}
\lambda_{22}=C_{66} \cos ^{2} \theta^{i}+C_{44} \sin ^{2} \theta^{i} \\
\lambda_{23}=0 \\
\lambda_{33}=C_{55} \cos ^{2} \theta^{i}+C_{33} \sin ^{2} \theta^{i} \\
\underset{\approx}{\boldsymbol{\lambda}}=\left[\begin{array}{cccc}
C_{11} \cos ^{2} \theta^{\prime}+C_{55} \sin ^{2} \theta^{\prime} & 0 & C_{13} \sin \theta^{\prime} \cos \theta^{\prime}+C_{55} \sin \theta^{\prime} \cos \theta^{\prime} \\
0 & C_{66} \cos ^{2} \theta^{\prime}+C_{44} \sin ^{2} \theta^{\prime} & 0 \\
C_{13} \sin \theta^{\prime} \cos \theta^{\prime}+C_{55} \sin \theta^{\prime} & 0 & C_{55} \cos ^{2} \theta^{i}+C_{33} \sin ^{2} \theta^{\prime}
\end{array}\right]
\end{gathered}
$$

The principal difficulty we encounter in solving the eigenvalue problem stems from the fact that the direction cosines of the refracted wave cannot be directly determined from Snell's law (as they can in an isotropic medium) because of the directional dependence of the wave velocities. While this complicates the situation, the problem may be solved numerically by noting that Snell's law, Equation (9), must still hold. Thus, we may represent

$$
\sin \theta^{i}=\frac{V_{i}}{V_{w}} \sin \theta^{i n}=K V_{i}
$$

for each wave and

$$
\cos \theta^{i}=\left(1-\left(\frac{V_{i}}{V_{w}} \sin \theta^{i n}\right)^{2}\right)^{1 / 2}=\left(1-\left(K V_{i}\right)^{2}\right)^{1 / 2}
$$

Then, the eigenvalue problem becomes

$$
\left[\begin{array}{ccc}
C_{11}\left[1-\left(K V_{1}\right)^{2}\right]+C_{55}\left[\left(K V_{i}\right)^{2}\right]-e V_{t}^{2} & 0 & \left(C_{13}+C_{55}\right)\left[1-\left(K V_{1}\right)^{2}\right]^{1 / 2} K V_{t} \\
0 & \left.C_{66}\left[1-\left(K V_{1}\right)^{2}\right]+C_{44}\left(K V_{1}\right)^{2}\right]-e V_{t}^{2} & 0 \\
\left(C_{13}+C_{53}\right) K V_{1}\left[1-\left(K V_{1}\right)^{2}\right]^{1 / 2} & 0 & C_{55}\left[1-\left(K V_{1}\right)^{2}\right]+C_{33}\left[\left(K V_{1}\right)^{2}\right]-\varrho V_{1}^{2}
\end{array}\right]=0
$$

The result is similar to the case investigated by Kriz and Stinchcomb [5] for normal incidence along a non-symmetry direction with one pure mode shear wave, one quasilongitudinal wave, and one quasitransverse wave resulting. Since the pure mode shear wave will not be excited in the experimental arrangement 
shown, we restrict attention to the characteristic equation of remaining two waves:

$$
\begin{aligned}
\left\{C_{11}\left[1-\left(K V_{i}\right)^{2}\right]\right. & \left.+C_{55}\left(K V_{i}\right)-\varrho^{2}\right\}\left\{C_{55}\left[1-\left(K V_{i}\right)^{2}\right]+C_{33}\left(K V_{i}\right)^{2}\right\} \\
& -\left\{\left(C_{13}+C_{55}\right)^{2}\left(K V_{i}\right)^{2}\left(1-\left(K V_{i}\right)^{2}\right)\right\}=0
\end{aligned}
$$

This is a simple equation for $V_{i}^{2}$ which may be solved numerically for the two real roots associated with wave propagation and their associated eigenvectors.

\section{Energy Flux Deviation}

Once the velocities associated with quasilongitudinal $(\mathrm{QL})$ and quasitransverse (QT) wave propagation have been determined, it is then possible to calculate the energy flux vector for each mode of propagation. It should be noted that, unlike propagation along a symmetry axis, the energy flux vector for wave propagation in an arbitrary direction does not coincide with the wave normal. The energy flux vector is given by

$$
E_{j}=-\sigma_{i j} \dot{u}_{i}
$$

Hence, calculation of the eigenvectors for each mode is required (Equation (1)). Then, the particle displacements become

$$
u_{i}=\alpha_{i} A e^{i[\omega t-k(\cos \theta) x+(\sin \theta) z]}
$$

The stresses are then determined from the linearly elastic constitutive equation for the composite. For an orthotropic medium, the direction cosines for the energy flux vector then become

$$
\begin{aligned}
\frac{E}{|E|} & =-\left(\begin{array}{c}
E_{1} /|E| \\
0 \\
E_{3} /|E|
\end{array}\right) \\
& =-\left(\begin{array}{c}
\frac{C_{11} \alpha_{1}^{2} \cos \theta+C_{13} \alpha_{1} \alpha_{3} \sin \theta+C_{55} \alpha_{1} \alpha_{3} \sin \theta+C_{55} \alpha_{3}^{2} \cos \theta}{|E|} \\
0 \\
\frac{C_{33} \alpha_{3}^{2} \sin \theta+C_{13} \alpha_{1} \alpha_{3} \cos \theta+C_{55} \alpha_{1}^{2} \sin \theta+C_{55} \alpha_{1} \alpha_{3} \cos \theta}{|E|}
\end{array}\right)
\end{aligned}
$$

where

$$
|E|=\sqrt{E_{1}^{2}+E_{3}^{2}}
$$




\section{Amplitude Considerations}

We may represent the particle displacements for the incident wave by modifying Equation (1).

$$
\underline{u}_{i n}=A_{0}\left(\begin{array}{c}
\cos \theta^{i n} \\
0 \\
\sin \theta^{i n}
\end{array}\right) e^{i k^{L}\left(\cos \theta^{i n} x+\sin \theta^{i n} z-\omega t\right)}
$$

Similarly, we have

$$
u_{r e}=A_{r}\left(\begin{array}{c}
-\cos \theta^{r e} \\
0 \\
\sin \theta^{r e}
\end{array}\right) e^{i k^{L}\left(-\cos \theta^{r e} x+\sin \theta^{r e} z-\omega t\right)}
$$

For wave propagation in the composite, we have similar expressions for the generated quasilongitudinal (or longitudinal) and quasitransverse (or transverse) waves:

$$
\underline{u}_{Q L}=A_{Q L}\left(\begin{array}{c}
\alpha_{1}^{Q L} \\
0 \\
\alpha_{3}^{Q L}
\end{array}\right) e^{i k_{Q L}\left(\cos \phi^{Q L} x+\sin \phi^{Q L} z-\omega t\right)}
$$

and

$$
\underline{u}_{Q T}=A_{Q T}\left(\begin{array}{c}
\alpha_{1}^{Q T} \\
0 \\
\alpha_{3}^{Q T}
\end{array}\right) e^{i k_{Q L}\left(\cos \theta^{Q T} x+\sin \theta^{Q T} z-\omega t\right)}
$$

It should be noted that the eigenvectors $\alpha^{Q L}$ and $\alpha^{Q^{T}}$ are perpendicular to one another even though these are, in general, not pure mode waves $\left(\alpha^{Q L} \cdot \ell^{Q T} \neq 1\right.$, $\alpha^{Q L} \cdot \ell^{Q T} \neq 0$ ). Then, in order to calculate the reflection and transmission coefficients at the interface, it is necessary to satisfy the three boundary conditions at the fluid-solid interface.

(1) Continuity of Normal Displacement

$$
\left.u_{\text {water }}\right|_{x=0}=\left.u_{\text {composste }}\right|_{x=0}
$$

or

$$
\begin{aligned}
& A_{0} \cos \theta^{i n} e^{i k_{L}\left(\sin \theta^{\left.i n_{z-\omega t}\right)}\right.}-A_{r} \cos \theta^{r e} e^{i k_{L}\left(\sin \theta^{r e t-\omega t}\right)} \\
& =A_{Q L} \alpha_{1}^{Q L} e^{i k_{L}\left(\sin \theta Q L_{-\omega t}\right)}+A_{Q T} \alpha_{1}^{O T} e^{i Q^{Q T}\left(\sin \theta^{T T_{-\omega t}}\right)}
\end{aligned}
$$

which leads to an expression of Snell's law for the composite as before as well as 
the relationship

$$
A_{0} \cos \theta^{i n}-A_{r} \cos \theta^{i n}=A_{Q L} \alpha_{1}^{Q L}+A_{Q T} \alpha_{1}^{Q T}
$$

(2) Continuity of Normal Stress

$$
\begin{gathered}
\sigma_{11 \text { (water })_{x=0}}=\sigma_{\left.11_{\text {(composite) }}\right|_{x=0}} \\
\lambda\left[\left.\frac{\partial}{\partial x}\left(u_{\text {in }}+u_{r e}\right)\right|_{x=0}+\left.\frac{\partial}{\partial z}\left(w_{\text {in }}+w_{r e}\right)\right|_{x=0}\right] \\
=C_{11}\left[\frac{\partial}{\partial x}\left(u_{Q L}+u_{Q T}\right)\right]+C_{13}\left[\frac{\partial}{\partial z}\left(w_{Q L}+w_{Q T}\right)\right]
\end{gathered}
$$

which (invoking Snell's law) becomes

$$
\begin{gathered}
\lambda k_{L}\left[\left(\cos ^{2} \theta^{i n}+\sin ^{2} \theta^{i n}\right) A_{0}+\left(\cos ^{2} \theta^{r e}+\sin ^{2} \theta^{r e}\right) A_{r}\right] \\
\quad=C_{11}\left[A_{Q L} \alpha_{1}^{Q L} k_{Q L} \cos \theta^{Q L}+A_{Q T} \alpha_{1}^{Q T} k_{Q T} \cos \theta^{Q T}\right] \\
+C_{13}\left[A_{Q L} \alpha^{Q L} k_{Q L} \sin \theta^{Q L}+A_{Q T} \alpha^{Q T} k_{Q T} \sin \theta^{Q T}\right]
\end{gathered}
$$

and

(3) Zero Transverse Stress

Since the fluid cannot support a shear stress,

$$
C_{55} \epsilon_{5}=C_{55}\left(\frac{\partial\left(u_{Q L}+u_{Q T}\right)}{\partial z}+\frac{\partial\left(w_{Q L}+w_{Q T}\right)}{\partial x}\right)=0
$$

or

$$
\begin{aligned}
K_{Q L} \alpha_{1}^{Q L} \sin \theta^{Q L} A_{Q L} & +k_{Q T} \alpha_{1}^{Q T} \sin \theta^{Q T} A_{Q T}+k_{Q L} \alpha^{Q L} \cos \theta^{Q L} A_{Q L} \\
& +k_{Q T} \alpha_{Q T}{ }^{Q T} \cos \theta^{Q T} A_{Q T}=0
\end{aligned}
$$

which yields three equations in three unknowns which can be solved for the reflection and transmission coefficients $\left(R=A_{\text {ref }} / A_{0}, T_{Q L}=A_{Q L} / A_{0}\right.$, and $\left.T_{Q T}=A_{Q T} / A_{0}\right)$.

\section{RESULTS AND DISCUSSION}

Wave propagation at oblique angles incidence was studied for a graphite-epoxy composite in a variety of configurations. The composite geometry is illustrated in Figure 3 with the fiber reinforcement in the $y-z$ plane. Results are presented for 


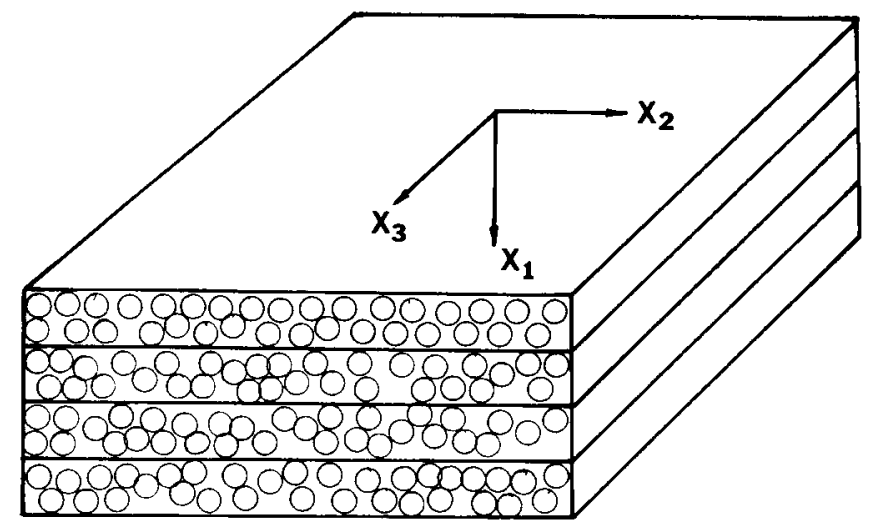

Figure 3. Coordinate system.

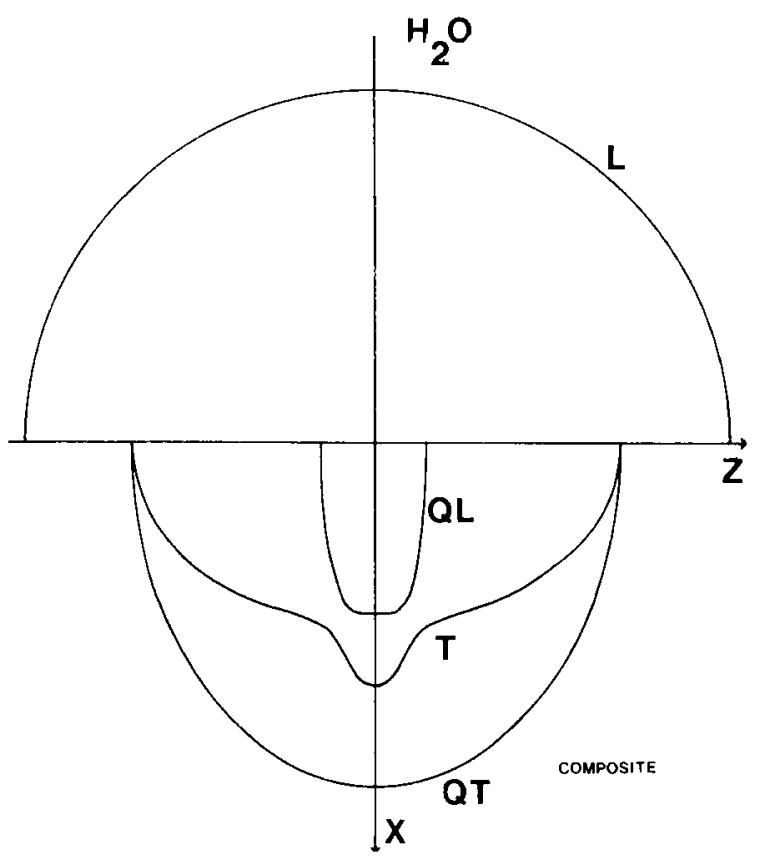

Figure 4. Section of slowness surface (parallel to fiber). 


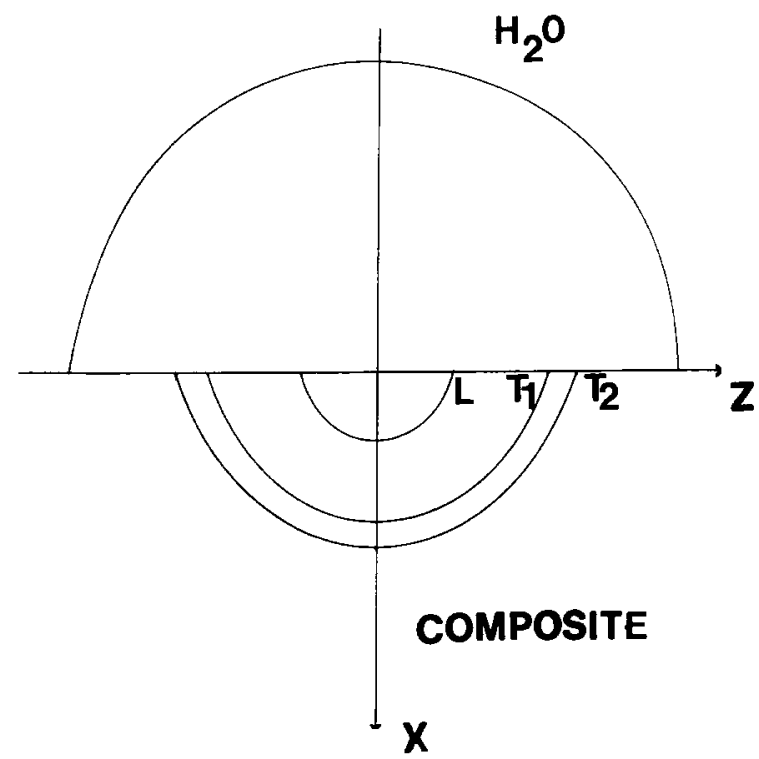

Figure 5. Section of slowness surface (perpendicular to fiber).

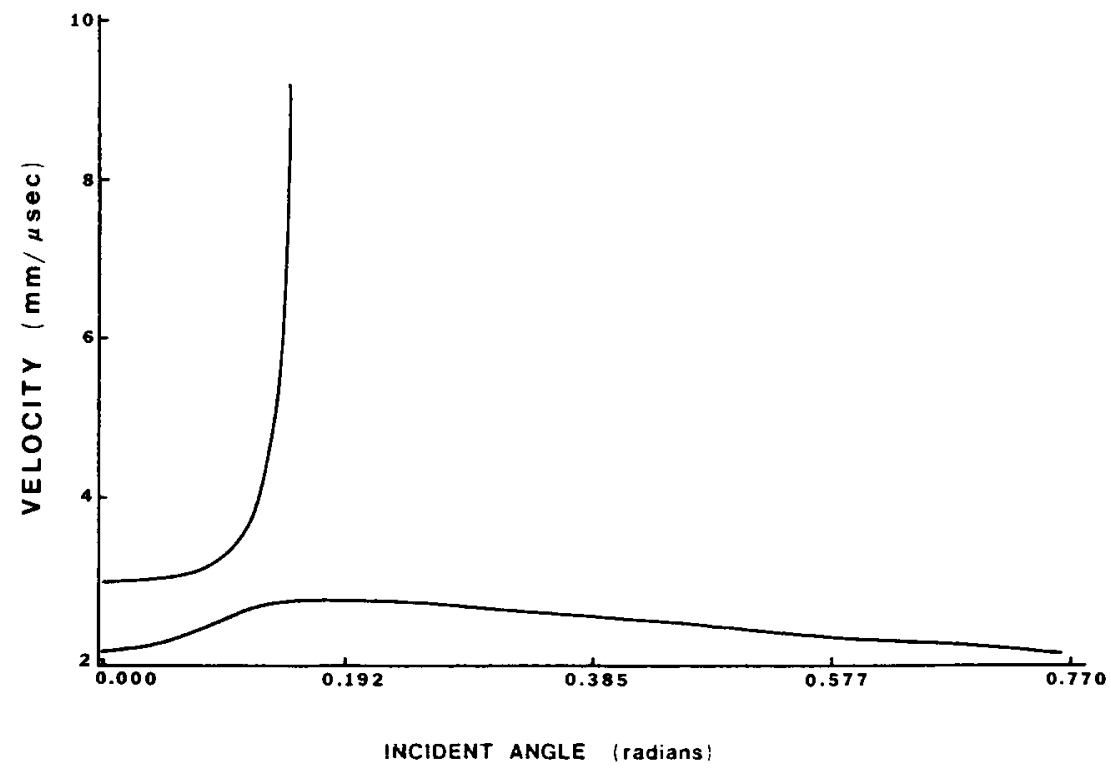

Figure 6. Phase velocity vs incident angle $\left(0^{\circ}\right)$. 


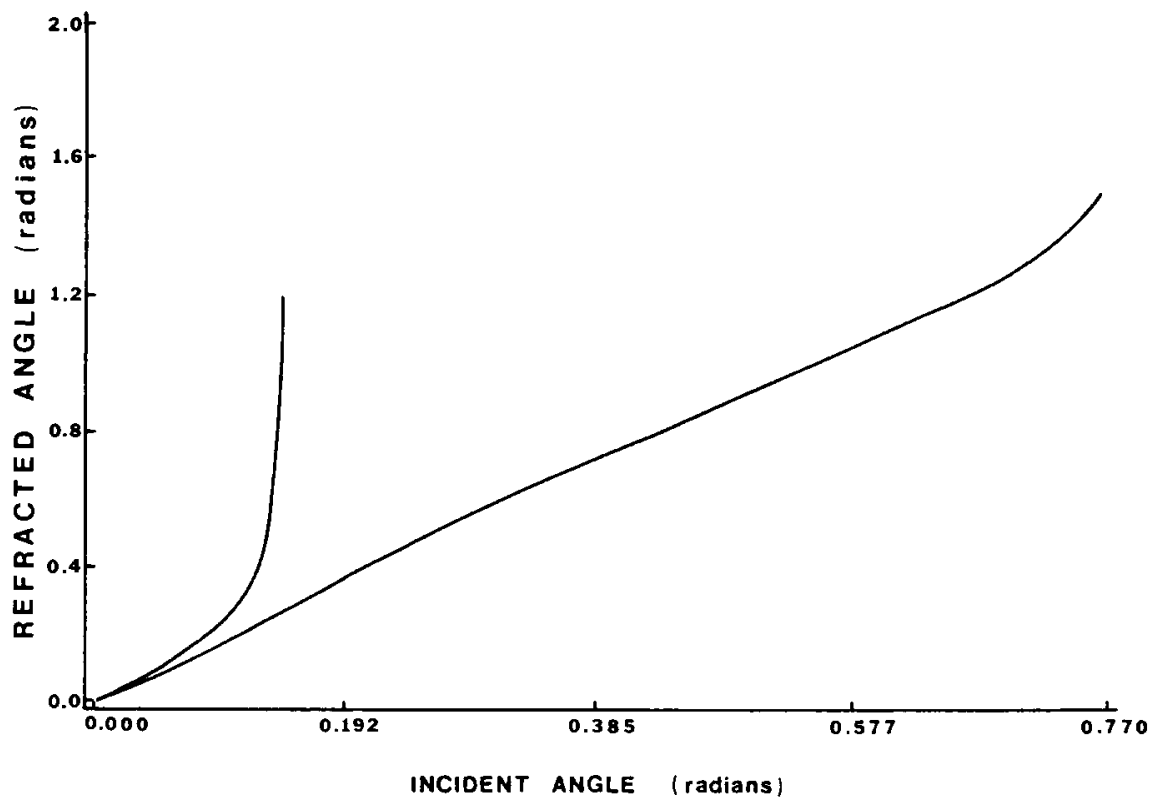

Figure 7. Wave normal vs incident angle $\left(0^{\circ}\right)$.

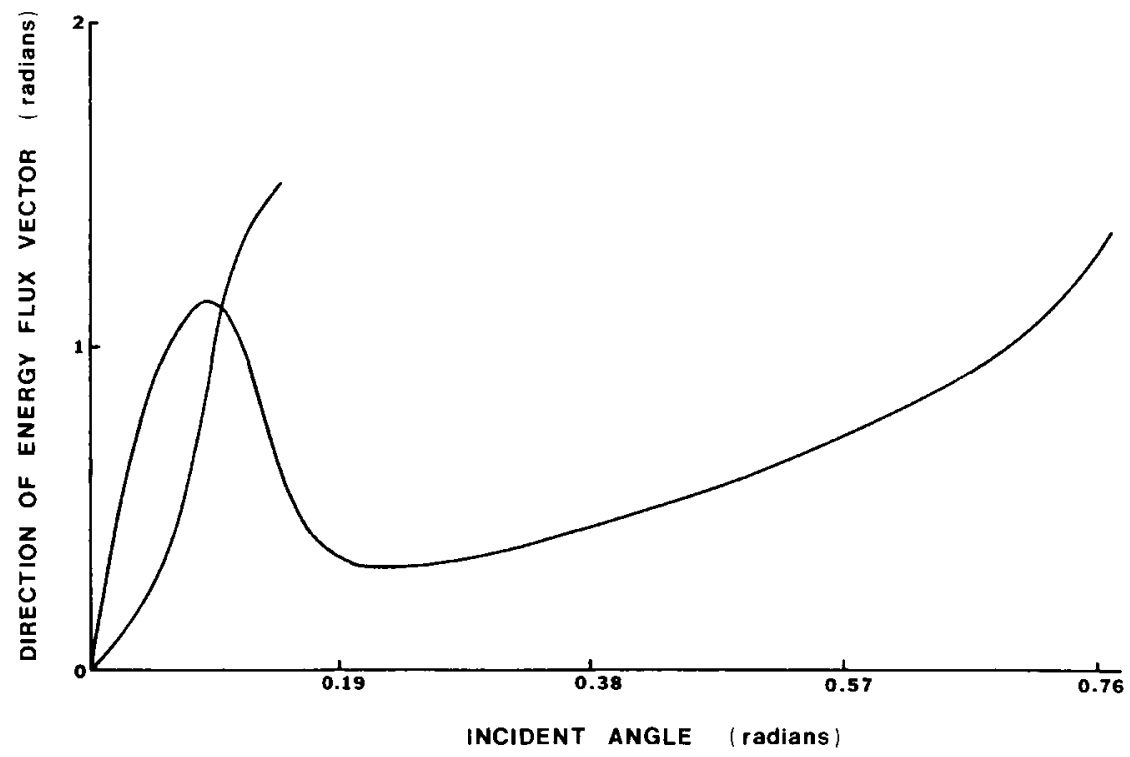

Figure 8. Energy flux vector vs incident angle $\left(0^{\circ}\right)$. 
a unidirectionally reinforced composite with the projection of the incident beam in the reinforcement plane (a) parallel, and (b) perpendicular to the fiber axis.

The slowness surface sections for the two cases under consideration here are presented in Figures 4 and 5. It is clear from the figures that both cases correspond to the usual situation with three possible transmitted waves. (However, it should be pointed out that the pure mode transverse wave in Figure 4 and one of the transverse waves in Figure 5 will not be excited with the experimental configuration under consideration). It is apparent in Figure 5 that the fiber axis is an axis of transverse isotropy with the section of the slowness surface for the longitudinal and shear waves being semicircular.

Results from the calculations are presented in Figures 6-10. For the parallel incidence case, the variations in velocity, refracted angle and energy flux vector of the generated quasilongitudinal and quasitransverse waves are presented. However, since the plane perpendicular to the fiber reinforcement is a plane of transverse isotropy, the waves generated for the $90^{\circ}$ case are pure mode longitudinal and shear waves with no velocity variation with the direction of the wave normal and no energy flux deviation. Accordingly, only the variation in the refracted angle with changing angle of incidence is presented in this case. The data used to characterize each ply of the graphite-epoxy composite are presented in Table 1. They are identical to those used by Kriz and Stinchcomb [5] and represent the typical behavior of a single composite ply.

One of the principal features of wave propagation in anisotropic media is the variation in velocity with propagation direction. This effect can be quite pronounced in graphite-epoxy laminates as is seen, for example, in Figure 6 for uni-

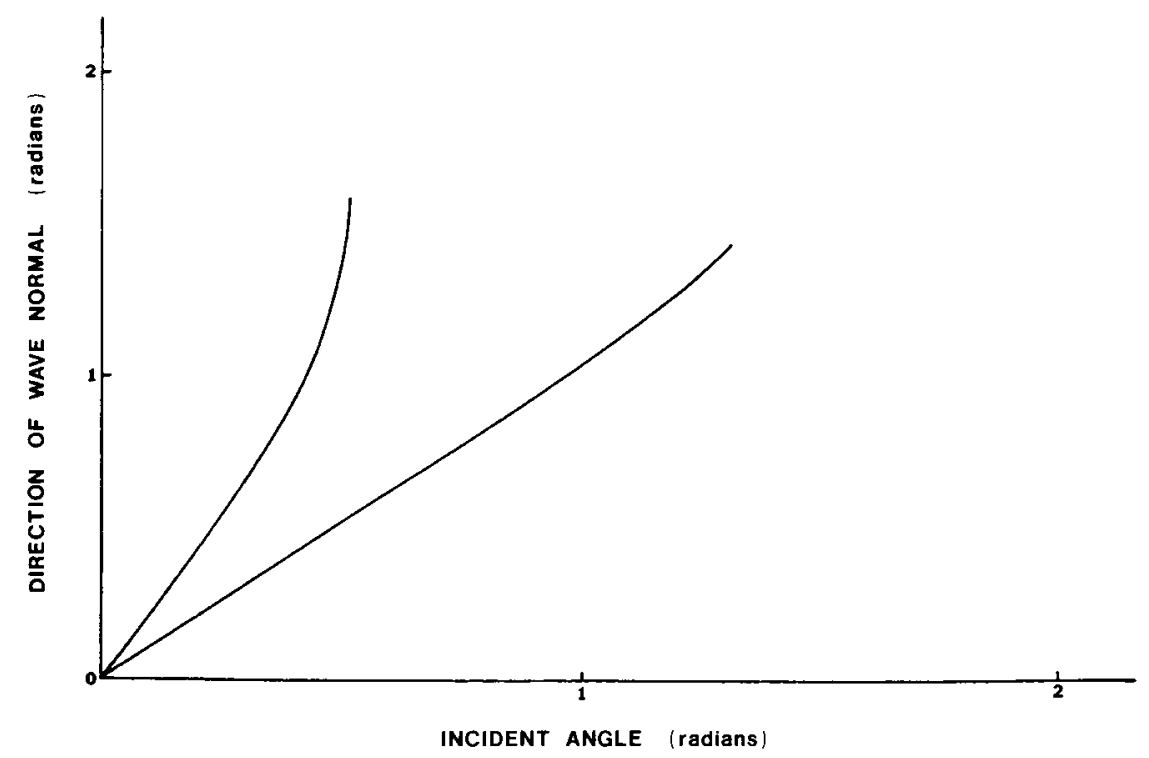

Figure 9. Wave normal vs incident angle $\left(90^{\circ}\right)$. 


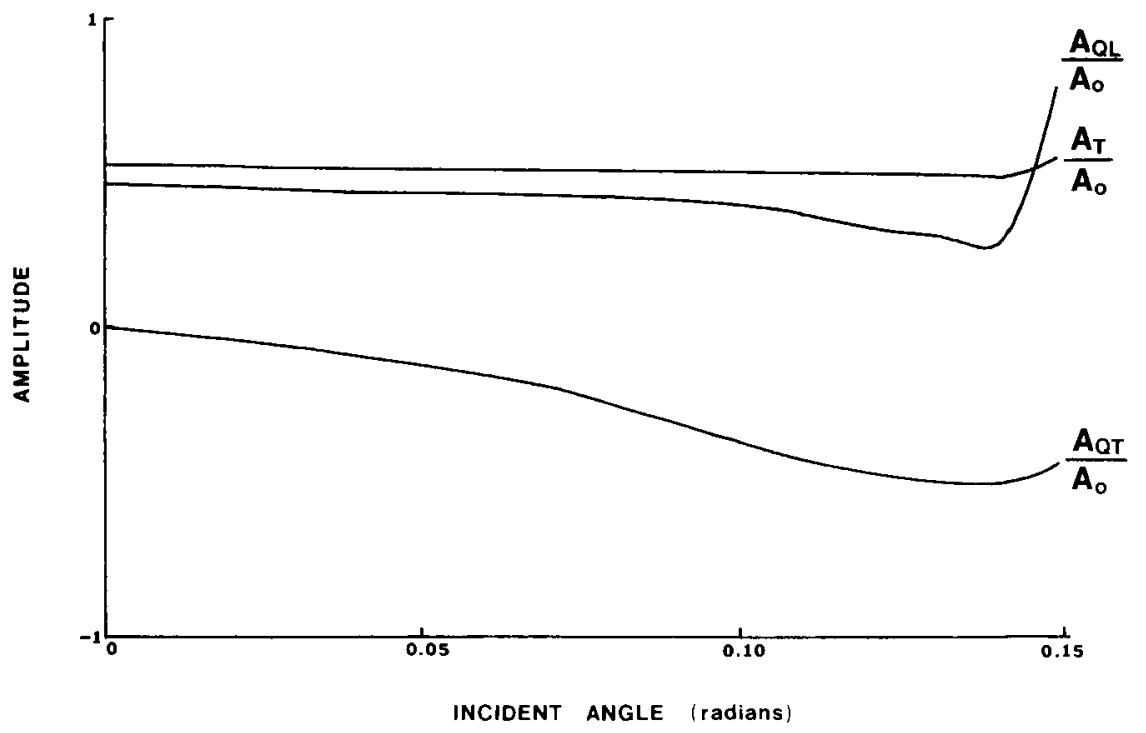

Figure 10. Amplitudes of reflected and transmitted waves vs incident angle $\left(0^{\circ}\right)$.

directional composite at $0^{\circ}$ incidence. It is interesting to note the precipitous rise in the velocity of the quasilongitudinal wave as the first critical angle is approached. This is attributable in large measure to the increasing contribution of the reinforcing fiber to the composite stiffness as the refracted angle approaches $90^{\circ}$. The behavior of the quasitransverse wave, however, is somewhat different in this respect. The variation observed in this case is much less than that of the quasilongitudinal wave with velccity increases to a maximum in the vicinity of the first critical angle and then decreases as the second critical angle is approached. It is not surprising that the variation in velocity of the quasitransverse wave should be appreciably less than that of the quasilongitudinal since the effect of the fibers on the stiffness of the composite in the direction perpendicular to the fiber axis should be much smaller. It is also interesting to note the large deviation in the energy flux vector from the wave normal in these composites (e.g. Figures 7 and 8). This is an important factor to consider if through transmission inspection is contemplated. Similar results are observed for the other configurations.

Figure 10 present plots of the reflected amplitude (for the longitudinal wave in

Table 1. (From Kriz and Stinchcomb [5]).

\begin{tabular}{ll}
$\mathrm{C}_{11}=\mathrm{C}_{22}=14.5 \mathrm{GPa}$ & $\mathrm{C}_{12}=7.24 \mathrm{GPa}$ \\
$\mathrm{C}_{33}=161 \mathrm{GPa}$ & $\mathrm{C}_{44}=\mathrm{C}_{55}=7.1 \mathrm{GPa}$ \\
$\mathrm{C}_{23}=\mathrm{C}_{13}=6.5 \mathrm{GPa}$ & $\mathrm{C}_{66}=\left(\mathrm{C}_{11}-\mathrm{C}_{12}\right) / 2=3.63 \mathrm{GPa}$ \\
\hline
\end{tabular}


water) as well as transmitted wave amplitudes (for the quasilongitudinal and quasitransverse waves in the composite) for the parallel incidence case. Here, calculations are shown for angles of incidence $\left(\theta^{i n}\right)$ increasing from normal incidence to the point where the boundary conditions at the interface can no longer be satisfied, i.e.

$$
\sin \theta^{Q L}=\frac{V_{Q L}}{V_{w}} \sin \theta^{i n}=1
$$

Beyond this angle of incidence,

$$
\frac{V_{i}}{V_{w}} \sin \theta^{i n}>1
$$

and there is no physically possible $\theta^{Q L}$ which will satisfy this condition. Hence, the boundary conditions cannot be solved with the formulation. It should also be pointed out that there is in general some ambiguity in determining the actual critical angle in an anisotropic medium as energy flux considerations must also be taken into account. Henneke and Jones [10] have proposed an alternative critical angle criterion based on the disappearance of a mode when its energy flux vector becomes parallel to the boundary. Discrepancies between the two criterion may arise, depending on the shape of the slowness surface. For the problems investigated in this study, no such ambiguity arises since $\underset{\sim}{E}|| \underset{\sim}{m}$ at the critical

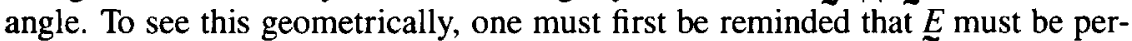
pendicular to the line tangent to the slowness surface at the point where the slowness vector intersects the slowness surface. Then, at the point where $\underset{\sim}{m}$ is parallel to the boundary $E$ must also be parallel to the boundary. This may not be the case for all possible composite configurations but is true for the cases studied here.

\section{CONCLUSIONS}

1. A microcomputer-based technique has been developed for studying wave propagation features in orthotropic materials for oblique angles of incidence.

2. This work demonstrates that, even though it may be somewhat more cumbersome to use oblique angles of incidence in composites than it is in isotropic media, the same mode conversion techniques can be applied and can yield important information about laminate properties.

3. It is expected that this approach may be used in a scanning mode to map out local variations in some or all orthotropic moduli. It should be noted that this will be useful from both a qualitative standpoint (e.g. rapid visual identification of region of substandard properties) as well as providing a quantitative measure of all pertinent elastic moduli.

\section{REFERENCES}

1. Kline, R. A. "The Origin of Localized Weak Areas in SMC Composites," Materials Evaluation, 40:874-879 (1982). 
2. Sachse, W. "Measurement of Elastic Moduli of Continuous-Filament and Eutectic Composite Materials," J. Composite Materials, 2:378-390 (1974).

3. Dean, G. D. and P. Turner. "The Elastic Properties of Carbon Fibers and Their Composites," Composites, 4:174-180 (1973).

4. Zimmer, J. E. and J. R. Cost. "Determination of the Elastic Constants of Unidirectional Fiber Composites Using Ultrasonic Velocity Measurements," J. Acoust. Soc. Am, 41:795-799 (1970).

5. Kriz, R. D. and W. W. Stinchcomb. "Elastic Moduli of Transversely Isotropic Graphite Fibers and Their Composites," Experimental Mechanics, 19:41-49 (1979).

6. Hosten, B. and B. Tittmann. "Elastic Anisotropy of Carbon-Carbon Composites During the Fabrication Process," in Proc., 1986 IEEE Symposium, Paper No. HH4, Williamsburg, VA (1986).

7. Musgrave, M. J. P. Proceedings of the Royal Society of London, A226:339-355 (1954).

8. Fedorov, F. I. Theory of Elastic Waves in Crystals, Plenum, New York (1968).

9. Henneke, E. G., II. "Reflection-Refraction of a Stress Wave at a Plane Boundary Between Anisotropic Media," J. Acoust. Soc. Am., 51:210-217 (1972).

10. Henneke, E. G., II and G. L. Jones. "Critical Angle for Reflection at a Liquid-Solid Interface in Single Crystals," J. Acoust. Soc. Am., 59:204-205 (1976).

11. Rokhlin, S., T. Bolland and L. Adler. "Reflection and Refraction of Elastic Waves on a Plane Interface Between Two Generally Anisotropic Media," J. Acoust. Soc. Am., 79:906-919 (1986). 